\title{
NATURALNESS RE-EXAMINED: IMPLICATIONS FOR SUPERSYMMETRY SEARCHES
}

\author{
J. L. FENG ${ }^{a \dagger}$, K. T. MATCHEV ${ }^{b *}$ and T. MOROI ${ }^{c \dagger}$ \\ ${ }^{\dagger}$ School of Natural Sciences, Inst. for Advanced Study, Princeton, NJ 08540, USA \\ * Theoretical Physics Department, Fermilab, Batavia, IL 60510, USA \\ E-mail: ${ }^{a}$ feng@ias.edu, ${ }^{b}$ matchev@fnal.gov, ${ }^{c}$ moroi@ias.edu \\ IASSNS-HEP-00/20, FERMILAB-Conf-00/062-T
}

\begin{abstract}
We discuss the origin of "focus points" in the scalar mass RGEs of the MSSM and their implications for collider searches. We present a new exact analytic solution to the homogeneous system of scalar mass RGEs in the MSSM for general $\tan \beta$. This is then used to prove that the focus point for $m_{H_{u}}^{2}$ depends only on the value of the top Yukawa coupling at the weak scale (not its value at the GUT scale) and is independent of the bottom Yukawa coupling.
\end{abstract}

\section{Focus Points in Supersymmetry}

Supersymmetry (SUSY) is the leading candidate for new physics beyond the Standard Model. It provides a framework for naturally explaining the stability of the weak scale with respect to radiative corrections. In its minimal version, the Minimal Supersymmetric Standard Model (MSSM), it also successfully predicts gauge coupling unification. Both of these attractive features of SUSY hinge on the assumption that the scale of the superpartner masses is not too far above the electroweak scale. This has reinforced a widespread optimism that the next round of collider experiments at the Tevatron, LHC or the NLC are guaranteed to discover all superpartners, if they exists.

In SUSY, the weak scale is determined by the relevant model parameters the soft SUSY breaking Higgs masses, $m_{H_{u}}$ and $m_{H_{d}}$, and the supersymmetric Higgs mass parameter, $\mu$ :

$$
\frac{1}{2} m_{Z}^{2}=\frac{m_{H_{d}}^{2}-m_{H_{u}}^{2} \tan ^{2} \beta}{\tan ^{2} \beta-1}-\mu^{2} \sim-m_{H_{u}}^{2}-\mu^{2},
$$

where the last relation holds for large $\tan \beta$. Naturalness requires that there be no large cancellations in the RHS of eq. (1) when expressed in terms of the fundamental parameters $\left\{a_{i}\right\}$ of the model (e.g., in minimal supergravity (mSUGRA), $\left\{a_{i}\right\}=\left\{m_{0}, M_{1 / 2}, \mu_{0}, A_{0}, B_{0}\right\}$ ). Otherwise an unwanted hierarchy is reintroduced, and the appeal of SUSY as a solution to the hierarchy problem is lost. The degree of fine-tuning iqvolved in eq. (11) is usually quantified in terms of the sensitivity coefficients $c_{a_{i}} \equiv\left|\partial \ln m_{Z}^{2} / \partial \ln a_{i}\right|$. 


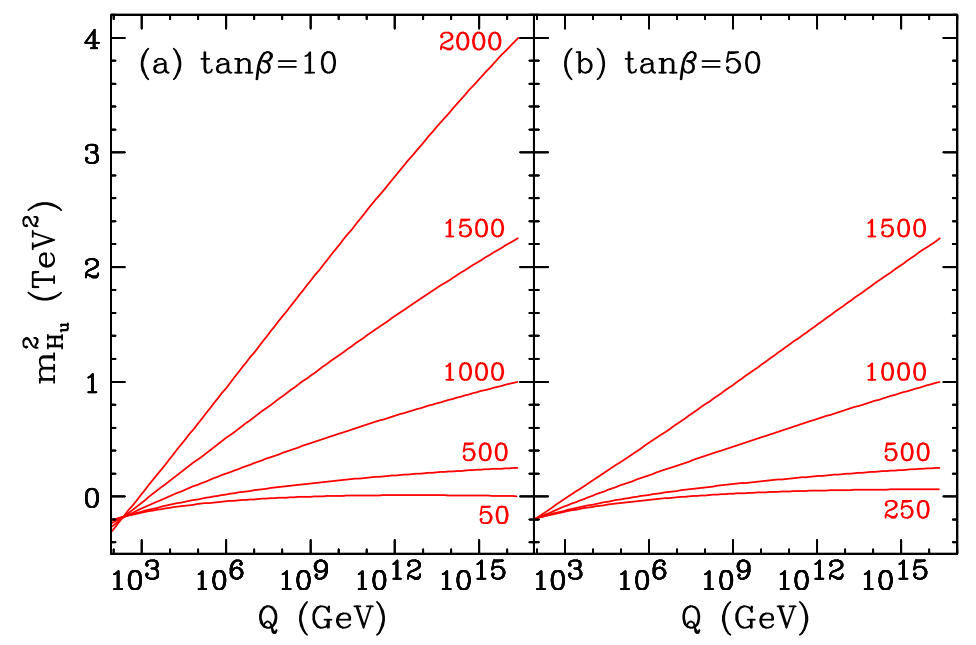

Figure 1: The RG evolution of $m_{H_{u}}^{2}$ for (a) $\tan \beta=10$ and (b) $\tan \beta=50$, several values of $m_{0}$ (shown, in $\mathrm{GeV}$ ), $M_{1 / 2}=300 \mathrm{GeV}, A_{0}=0$, and $m_{t}=174 \mathrm{GeV}$.

Recently we pointed out 2 that a general class of supersymmetric theories, including mSUGRA, exhibits "focus points" in the MSSM renormalization group equations (RGEs). (See also Ref. interesting range of top quark mass values, $m_{t} \sim 170-180 \mathrm{GeV}, m_{H_{u}}$ has such a focus point at the weak scale and is therefore highly insensitive to its GUT scale boundary value $m_{0}$ (see Fig. 11).

The focus point for $m_{H_{u}}^{2}$ implies that $c_{m_{0}}$ is small 2 . In Fig. 2 2 we show contours of the overall sensitivity parameter $c \equiv \max \left\{c_{a_{i}}\right\}$. We see that regions of parameter space with $m_{0} \sim 2-3 \mathrm{TeV}$ are as natural as regions with $m_{0} \lesssim 1 \mathrm{TeV}$. Hence, it is quite possible that all squarks and sleptons have multi-TeV masses, making their discovery at colliders significantly more challenging than conventionally expected. On the other hand, Fig. 2 reveals that naturalness restricts the gaugino mass $M_{1 / 2}$ to the few hundred $\mathrm{GeV}$ range.

The focus point mechanism is surprisingly robust against variations of the input parameters, e.g., $M_{1 / 2}, A_{0}, m_{t}$ and $M_{\mathrm{GUT}}$. We will prove analytically that the focus point scale is also independent of $\tan \beta$. This supplements previous numerical demonstrations of this result 2 . In the process (see section 2) we shall derive analytic solutions to the homogeneous parts of the scalar mass RGEs for arbitrary values of $\tan \beta$. To our knowledge, such solutions

${ }^{a}$ We are grateful to Jon Bagger for stimulating questions and discussions. 


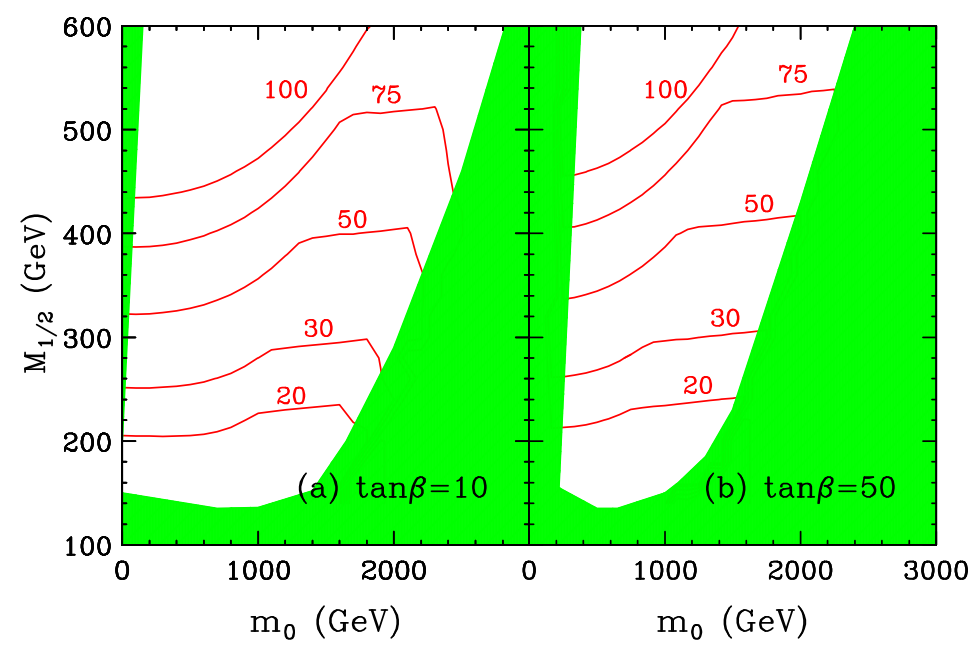

Figure 2: Contours of constant sensitivity parameter $c$ in the $\left(m_{0}, M_{1 / 2}\right)$ plane for (a) $\tan \beta=10$ and (b) $\tan \beta=50, A_{0}=0, \mu>0$, and $m_{t}=174 \mathrm{GeV}$. The bottom and right shaded region is excluded by the chargino mass limit of $90 \mathrm{GeV}$. The top left region is also excluded if a neutral LSP is required.

have not been presented previously in the literature.

\section{Analytic Solution to the Homogeneous System of Scalar RGEs}

Neglecting the tau Yukawa coupling and the hypercharge differences for top and bottom, the RGEs for the relevant Yukawa couplings read

$$
\dot{Y}_{t}=Y_{t}\left(6 Y_{t}+Y_{b}-a_{i} g_{i}^{2}\right), \quad \dot{Y}_{b}=Y_{b}\left(6 Y_{b}+Y_{t}-a_{i} g_{i}^{2}\right),
$$

where $Y_{t} \equiv y_{t}^{2}, Y_{b} \equiv y_{b}^{2}, 8 \pi^{2} t \equiv \ln Q$ and ${ }^{*} \equiv d / d t$. The homogeneous system of RGEs for the relevant scalar masses is

$$
\dot{\vec{M}}=\mathbf{N} \vec{M}
$$

where $\vec{M}^{T}=\left(m_{H_{u}}^{2}, m_{U}^{2}, m_{Q}^{2}, m_{D}^{2}, m_{H_{d}}^{2}\right)$ and

$$
\mathbf{N}=Y_{t}\left(\begin{array}{lllll}
3 & 3 & 3 & 0 & 0 \\
2 & 2 & 2 & 0 & 0 \\
1 & 1 & 1 & 0 & 0 \\
0 & 0 & 0 & 0 & 0 \\
0 & 0 & 0 & 0 & 0
\end{array}\right)+Y_{b}\left(\begin{array}{lllll}
0 & 0 & 0 & 0 & 0 \\
0 & 0 & 0 & 0 & 0 \\
0 & 0 & 1 & 1 & 1 \\
0 & 0 & 2 & 2 & 2 \\
0 & 0 & 3 & 3 & 3
\end{array}\right)
$$


Now perform a suitable change of variables

$$
\left(\begin{array}{c}
m_{H_{u}}^{2} \\
m_{U_{3}}^{2} \\
m_{Q_{3}}^{2} \\
m_{D_{3}}^{2} \\
m_{H_{d}}^{2}
\end{array}\right)=\left(\begin{array}{rrrrr}
1 & 3 & 0 & 0 & 0 \\
-1 & 2 & 1 & 0 & 0 \\
0 & 1 & -1 & 1 & 0 \\
0 & 0 & 1 & 2 & 1 \\
0 & 0 & 0 & 3 & -1
\end{array}\right)\left(\begin{array}{c}
c_{0} \\
c_{t}(t) \\
c_{0}^{\prime} \\
c_{b}(t) \\
c_{0}^{\prime \prime}
\end{array}\right)
$$

which amounts simply to decomposing $\vec{M}$ into the eigenvectors of $\mathbf{N}$ (those are just the columns of the $5 \times 5$ matrix in the RHS of eq. (50). Since $\mathbf{N}$ has three zero eigenvalues, three of the new variables, namely $c_{0}, c_{0}^{\prime}$ and $c_{0}^{\prime \prime}$, are constants and do not run. We have thus reduced the system of five equations (3) to a system of two equations for the remaining new variables $c_{t}$ and $c_{b}$ :

$$
\dot{c}_{t}=Y_{t}\left(6 c_{t}+c_{b}\right), \quad \dot{c}_{b}=Y_{b}\left(6 c_{b}+c_{t}\right) .
$$

The GUT scale boundary conditions (BC) are

$$
c_{t}\left(t_{\mathrm{G}}\right)=k_{t}, \quad c_{b}\left(t_{\mathrm{G}}\right)=k_{b},
$$

where $k_{t}$ and $k_{b}$ are some model-specific pure numbers. In the absence of a general method for solving the system (6), we make the following ansatz:

$$
c_{t}(t)=k_{t}+Y_{t}(t) f_{t}(t), \quad c_{b}(t)=k_{b}+Y_{b}(t) f_{b}(t),
$$

with $f_{t}\left(t_{\mathrm{G}}\right)=f_{b}\left(t_{\mathrm{G}}\right)=0$. Then, we get

$$
\dot{f}_{t}=6 k_{t}+k_{b}+a_{i} g_{i}^{2} f_{t}-Y_{b}\left(f_{t}-f_{b}\right),
$$

and $\dot{f}_{b}$ is given by replacing the subscript $t \leftrightarrow b$. Now, by calculating $\dot{f}_{t}-\dot{f}_{b}$, we find a simple equation for $f_{t}-f_{b}$,

$$
\left(\dot{f}_{t}-\dot{f}_{b}\right)+\left(Y_{t}+Y_{b}-a_{i} g_{i}^{2}\right)\left(f_{t}-f_{b}\right)=5\left(k_{t}-k_{b}\right),
$$

which can be easily integrated. To simplify notation, let us define

$$
Y(t) \equiv \int_{t_{G}}^{t} d t^{\prime}\left(Y_{t}\left(t^{\prime}\right)+Y_{b}\left(t^{\prime}\right)\right), \quad G(t) \equiv \int_{t_{G}}^{t} d t^{\prime} a_{i} g_{i}^{2}\left(t^{\prime}\right) .
$$

Then the solution to eq. (10) can be written as

$$
f_{t}(t)-f_{b}(t)=5\left(k_{t}-k_{b}\right) e^{G(t)-Y(t)} \int_{t_{G}}^{t} d t^{\prime} e^{-G\left(t^{\prime}\right)+Y\left(t^{\prime}\right)},
$$


where we have fixed the integration constant using $k_{t}=k_{b}$. It is now trivial to solve for $f_{t}(t)$ and $f_{b}(t)$ themselves. The final solution for $c_{t}(t)$ is

$$
\begin{aligned}
c_{t}(t)= & k_{t}+Y_{t}(t) e^{G(t)}\left\{\left(6 k_{t}+k_{b}\right) \int_{t_{G}}^{t} d t^{\prime} e^{-G\left(t^{\prime}\right)}\right. \\
& \left.-5\left(k_{t}-k_{b}\right) \int_{t_{G}}^{t} d t^{\prime} Y_{b}\left(t^{\prime}\right) e^{-Y\left(t^{\prime}\right)} \int_{t_{G}}^{t^{\prime}} d t^{\prime \prime} e^{Y\left(t^{\prime \prime}\right)-G\left(t^{\prime \prime}\right)}\right\} .
\end{aligned}
$$

The solution for $c_{b}(t)$ is obtained simply by interchanging $t \leftrightarrow b$ in (13). As analytic solutions for $Y_{t}$ and $Y_{b}$ are known $\theta$, this then completes the analytic solution of the homogeneous scalar mass RGEs for arbitrary $\tan \beta$.

Now, we discuss the $\tan \beta$ independence of the focus point scale of $m_{H_{u}}^{2}$. Define the focus point scale $Q_{\mathrm{F}}^{\left(H_{u}\right)}$ as

$$
\left.\frac{d m_{H_{u}}^{2}}{d m_{0}}\right|_{Q=Q_{\mathrm{F}}^{\left(H_{u}\right)}}=0 .
$$

The $m_{0}$ dependence of $m_{H_{u}}^{2}$ can be studied by using the homogeneous part of the RGE for the squared scalar masses, and hence $Q_{\mathrm{F}}^{\left(H_{u}\right)}$ is obtained by solving the following equation:

$$
c_{0}+3 c_{t}\left(t_{F}\right) \equiv 0,
$$

where $8 \pi^{2} t_{F}=\ln Q_{\mathrm{F}}^{\left(H_{u}\right)}$. In mSUGRA we find $c_{0}=-\frac{2}{7} m_{0}^{2}$, while $c_{t}\left(t_{F}\right)$ is given by eq. (13) with $t=t_{F}$ and $k_{t}=k_{b}=\frac{3}{7} m_{0}^{2}$ :

$$
c_{t}\left(t_{F}\right)=\left(\frac{3}{7}+3 Y_{t}\left(t_{F}\right) e^{G\left(t_{F}\right)} \int_{t_{G}}^{t_{F}} d t^{\prime} e^{-G\left(t^{\prime}\right)}\right) m_{0}^{2} .
$$

The boundary condition $k_{t}=k_{b}$ leads to an extreme simplification, as the second term in the brackets in eq. (13) vanishes. Substituting (16) into eq. (15), we obtain the focus point condition in the form

$$
Y_{t}\left(t_{F}\right) e^{G\left(t_{F}\right)} \int_{t_{F}}^{t_{G}} d t^{\prime} e^{-G\left(t^{\prime}\right)}=\frac{1}{9}
$$

The focus point may therefore be written in terms of only the gauge couplings and $y_{t}\left(t_{F}\right)$. The extremely mild $\tan \beta$ dependence of $Q_{\mathrm{F}}^{\left(H_{u}\right)}$ for moderate to large $\tan \beta$ then follows from $y_{t}\left(t_{F}\right) \propto 1 / \sin \beta \sim$ const $+\mathcal{O}\left(\tan ^{-2} \beta\right)$. Note that as $\tan \beta$ varies in this range, $y_{b}$ and $y_{t}\left(t_{G}\right)$ will vary. The variation in $y_{t}\left(t_{G}\right)$ was claimed in Ref. 5 to destroy the insensitivity of $m_{H_{u}}$ to $m_{0}$. We see, however, that this variation is irrelevant for the focus point: the focus point depends on $y_{t}\left(t_{F}\right)$, not $y_{t}\left(t_{G}\right)$, and for all moderate and large $\tan \beta$, the focus point remains at the weak scale, and the sensitivity coefficient $c_{m_{0}}$ is small. 


\section{Implications For Supersymmetry Searches}

We have seen that the naturalness bound on $m_{0}$ (i.e., the typical sfermion mass) may be as large as a few $\mathrm{TeV}$ (see Fig. 2). This result has important implications for the superpartner spectrum and, in particular, the discovery prospects for scalar superpartners at future colliders.

Multi-TeV sleptons (whose masses scale as $\sim m_{0}$ ), for example, are beyond the kinematic limit $\sqrt{s} / 2$ of all proposed linear colliders and will also escape detection at hadron colliders, as they are not strongly produced, and will rarely be obtained in the cascade decays of strongly interacting superparticles. Multi$\mathrm{TeV}$ squarks will, of course, also evade proposed linear colliders. They will also stretch the LHC reach, although a recent study 6 in the lepton plus jets channel has revealed discovery potential even for squarks up to $3 \mathrm{TeV}$.

In contrast to the sfermions, gauginos and higgsinos cannot be very heavy in this scenario. For example, the constraint $c \leq 50$ implies $M_{1 / 2} \lesssim 400 \mathrm{GeV}$,

corresponding to $M_{1} \lesssim 160 \mathrm{GeV}, M_{2} \lesssim 320 \mathrm{GeV}$, and $M_{3} \lesssim 1.2 \mathrm{TeV}$. Such gauginos will be produced in large numbers at the LHC, and will be discovered in typical scenarios. (However, the detectability of all gauginos and higgsinos in cases of mass degeneracies at the LHC remains an open question.)

Finally, in spite of the relatively heavy squark masses allowed by the focus point mechanism, the most natural range for the light Higgs mass is somewhere below $118 \mathrm{GeV}$. This is because large values of $\left|A_{0}\right|$ (and hence large stop mixing) are disallowed by naturalness, as they induce too large $m_{H_{u}}^{2}$ through the RGEs. Therefore, even if all sleptons and squarks are very heavy, Run II of the Tevatron has a golden opportunity to explore much of the most natural mSUGRA parameter space in its search for the lightest Higgs boson.

\section{Acknowledgments}

This work was supported in part by the Department of Energy under contracts DE-FG02-90ER40542 and DE-AC02-76CH03000, by the National Science Foundation under grant PHY-9513835, through the generosity of Frank and Peggy Taplin (JLF), and by a Marvin L. Goldberger Membership (TM).

\section{References}

1. J. Ellis, K. Enqvist, D. V. Nanopoulos and F. Zwirner, Mod. Phys. Lett. A1, 57 (1986); R. Barbieri and G. F. Giudice, Nucl. Phys. B306, 63 (1988).

2. J. L. Feng, K. T. Matchev and T. Moroi, Phys. Rev. Lett. 84, 2322 (2000); Phys. Rev. D61, 075005 (2000); hep-ph/9909334. 
3. J. L. Feng and T. Moroi, hep-ph/9907319; K. Agashe, hep-ph/9910497.

4. E. G. Floratos and G. K. Leontaris, Phys. Lett. B336, 194 (1994).

5. A. Romanino and A. Strumia, hep-ph/9912301.

6. B. C. Allanach et al., hep-ph/9912302. 